\section{No CFTR: are CF symptoms milder?}

Sir - Cystic fibrosis (CF) is a recessive disease caused by mutations in a single gene ${ }^{1}$ that codes for the cystic fibrosis transmembrane conductance regulator (CFTR), a small conductance, cAMP-activated $\mathrm{Cl}^{-}$channel $l^{2,3}$. Many mutations that cause CF, including the most common mutation $(\Delta \mathrm{F} 508)$, lead to abnormal processing of CFTR ${ }^{4}$, and most symptoms of $\mathrm{CF}$ are linked to decreased plasma membrane $\mathrm{Cl}^{-}$conductance ${ }^{5}$. How do having a milder form of $\mathrm{CF}^{7,8}$. One of the earlier homozygous stop codon patients ing defect ${ }^{10}$. In short, the surprising notion that $\Delta \mathrm{F} 508$ mutations are worse than the complete absence of CFTR protein has caught on ${ }^{4,6-10}$.

However, the disruptive effects proposed for $\Delta F 508$ should give rise to a dominant rather than recessive pattern of inheritance ${ }^{4,6}$. Because $\mathrm{CF}$ is a recessive disease, the conclusion that missense mutations are worse than nonsense mutations bears close scrutiny.

A large study of CF subjects has established that $\triangle \mathrm{F} 508$ homozygotes are almost always pancreatic insufficient and have greatly variable lung function, which is, on average, poorer

Table 1 Selected features of CF patients with missense and nonsense mutations

\begin{tabular}{|c|c|c|c|c|c|}
\hline Mutation & $n$ & Age(yr) & Sweat $\mathrm{Cl}^{-}(\mathrm{mM})$ & $\mathrm{aFEV}_{1}$ & Ref. \\
\hline R553X/W1316X & 1 & 21 & 116 & 82 & 6 \\
\hline G542X/S1255X & $\uparrow$ & 11 & 114 & 66 & 6 \\
\hline G542X/G552X & 1 & 12.5 & positive & 120 & 7 \\
\hline R553X/R553X & 1 & 13.5 & 160 & mod. affected & 8 \\
\hline W1282XW1282X & 16 & $9.3 \pm 7.5$ & 113 & $64 \pm 27$ & 12 \\
\hline G542X/W1282X & 1 & 16 & - & 63 & 12 \\
\hline$\Delta \mathrm{F} 508 / \Delta \mathrm{F} 508$ & 149 & $17 \pm 10$ & 106 & $76-62^{b}$ & 11 \\
\hline
\end{tabular}

$n=11>$ age 6

$\%$ of predicted value

approximate values for linear regression at ages 10 and 20 respectively

different alleles affect phenotype, and what can this tell us about CFTR function?

In an early attempt to relate specific alleles with phenotype, two African-American CF patients who were homozygous for CFTR stop mutations were reported to have milder pulmonary symptoms than $\Delta \mathrm{F} 508$ homozygotes ${ }^{6}$, suggesting that $a b-$ sence of CFTR protein may be less deleterious than altered CFTR protein ${ }^{6}$. Shortly after that report, processing of $\triangle$ F508 CFTR (and several other missense mutations) was found to be grossly abnormal in cultured cells expressing recombinant $\triangle \mathrm{F} 508 \mathrm{CFTR}^{4}$. It was concluded that misprocessing of CFTR is the basis of most $\mathrm{CF}^{4}$, and that protein harbouring a missense mutation might retain partial activity while trapped at incorrect cellular locations, causing a more general dysfunction than complete absence of protein ${ }^{4}$. Two more CF patients were found to be homozygous for stop mutations, and were interpreted as than it is for pancreatic sufficient patients $^{11}$. The positive correlation between function of pancreas and lungs holds for $\triangle F 508$ heterozygotes and CF subjects homozygous for non$\Delta$ F508 mutation $^{11}$. The variation in lung function for $\Delta \mathrm{F} 508$ homozygotes is very large. For example, one measure of lung function, forced expiratory volume in $1 \mathrm{~s}\left(\mathrm{FEV}_{1}\right)$, ranged from $\sim 12-120 \%$ of that expected in the 10-15 age group ${ }^{11}$. This large variance in patients homozygous for one allele establishes the importance of factors other than CF genotype in the pathophysiology of CF lung disease. The four subjects homozygous for stop codons were $11,12.5,13.5$ and 21 years old (see Table) and have all been pancreatic insufficient since infancy. At least two are colonized with Pseudomonas aeruginosa, and all have clear signs of pulmonary dysfunction. $\mathrm{FEV}_{1}$ scores were and 66,82 , and $120 \%$ of expected values, and "moderately affected". These values lie within the distribution of pulmonary dysfunction for $\Delta \mathrm{F} 508$ homozygotes ${ }^{11}$. Recently, a sample of 18 individuals homozygous for CFTR stop mutations was discovered among CF patients of Ashkenazi Jewish origin. These patients have severe pulmonary disease $\mathrm{e}^{12}$. Taken together, there is no basis for claiming a significant difference in clinical phenotype between patients with nonsense mutations and $\Delta \mathrm{F} 508$ homozygotes. Importantly, all patients with nonsense mutations and all but $2 \Delta$ F508 subjects from ref. 11 (not included in the table) are pancreatic insufficient.

In conclusion, present evidence suggests that many CFTR mutations have a common phenotype which seems traceable to reduced cAMPactivated $\mathrm{Cl}^{-}$conductance. Whether loss of $\mathrm{Cl}$ conductance occurs because the mutant CFTR channels are absent, reduced in amount and open probability $^{14}$, or completely nonconducting appears to have less consequence for clinical status than other genetic and environmental factors. A smaller set of CFTR mutations is associated with pancreatic sufficiency, milder pulmonary disease, and improved sweat gland function $^{11,13}$. The $\mathrm{Cl}^{-}$impermeability hypothesis of CF predicts that these should be associated with residual CFTR $\mathrm{Cl}^{-}$channel function, and preliminary evidence supports that prediction ${ }^{15}$. This conclusion is consistent with the recessive nature of CF. It also means that gene or protein replacement therapies for $\mathrm{CF}$ should be effective on their own, without requiring concomitant silencing of the $\Delta \mathrm{F} 508$ gene.

Jeffrey J. Wine

Cystic Fibrosis Research

Laboratory,

Stanford University, Stanford,

California 94305-2130, USA

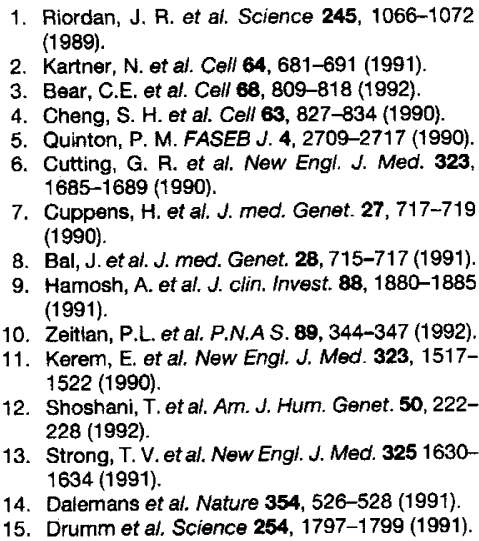

